\title{
Cold Pack Accelerate Recovery in Functional Parameters Post Ischemia Reperfusion Injury in Skeletal Muscle
}

\author{
Magda Carolina Diaz ${ }^{1}$, Karen Lizeth Álvarez Raigoza ${ }^{1}$, Saveria Sangiovanni González ${ }^{1}$, Leonardo Fierro² and Camilo \\ Morales Jiménez*1
}

${ }^{1}$ Pontificia Universidad Javeriana, Department of Basic Sciences of the Health, Group of Investigation in Basic Sciences and Clinics of Health, Cali-Colombia

${ }^{2}$ Department of Physiological Sciences, Faculty of Health, Univalle Pharmacology Research Group

Received: 些: October 25, 2018; Published: 制: November 14, 2018

*Corresponding author: Camilo Morales Jiménez, Department of Basic Health Sciences, Research Group in Basic Sciences and Clinics of Health, Colombia

\begin{abstract}
To determine the effect of a 30-minute application of cold pack in soleus muscle in muscular force and fatigue after 1,3 , and 7 days after subjection to 2 hours of ischemia. An in-vivo experimental study was performed using forty-nine rats were randomly divided into seven groups (control and 1,3, and 7 days post-ischemia with and without a cold pack). A 2-hour period of ischemia was performed on the lower extremity, followed by a 30-min application of a cold pack. After a one-day reperfusion, the group without and with cold pack application presented reduce on maximal rate of contraction round $40 \%$, on the fatigue time in $51 \%$ and on fatigue in repeated contraction in $50 \%$ in the first minute. After three days, the group without cold pack presented drop 50\% on maximum force and on the maximal rate of contraction and drop $90 \%$ in the fatigue in repeated contraction while with cold pack presented recovery in the maximal rate of contraction and the fatigue in repeated contraction in the second minute. After a seven-day reperfusion, the groups without and with cold pack application present recovery in all parameter except in the fatigue time. These results suggest that for ischemia-reperfusion that is produced in surgical situations involving the use of pneumatic tourniquets, the use of cold pack accelerate the recovery in functional parameters post-ischemia.
\end{abstract}

Keywords: Cryotherapy; Ischemia Reperfusion Injury; Muscle; Skeletal

\section{Introduction}

Skeletal muscle injury is the main cause of functional limitations in workers, as seen in occupational and sports medicine [1]. Within this group, muscle ischemia-reperfusion injury is present in diverse clinical situations such as peripheral arterial occlusive disease $[2,3]$, surgical situations involving the use of pneumatic tourniquets [4,5], muscle trauma compromising the vascular system and transplant or reconstructive surgery [6]. Histological studies of ischemia-reperfusion injury in skeletal muscle have shown edema, microvascular dysfunction, inflammation, a decrease in muscle fiber diameter and loss of angular contours, all of which are associated with morbidity and the dysfunction of skeletal muscle [7-11]. Moreover, studies of muscle function under isometric conditions show that maximum tetanic force and contraction rate in oxidative and glycolytic muscle drops during ischemia, and vastly recover throughout the first hour of reperfusion [12-14]. However, as the ischemic period extends, all functional parameters decline in both oxidative and glycolytic muscle groups; in fact, after two hours of ischemia, those functional parameters decrease to 80 and $90 \%$ of the values found before ischemia $[12,13]$. Cryotherapy is one of the main therapies used in the acute stages, along with compression and elevation, since its effect on arterial and venous vasoconstriction leads to a decreased blood flow to the treated area, thus reducing both edema and inflammation induced by trauma [15-19]. In skeletal muscle injury the effect of cryotherapy report is attenuates microcirculatory impairment, regional inflammation, and muscle necrosis [20,21]. Nevertheless, and in contrast with the advantages of cryotherapy in muscular injury, a different study showed that the use of 20 minutes of cryotherapy produces a delay in the differentiation of satellite cells in early stages of injury, thus provoking a setback in the regeneration of muscle fibers, yielding much smaller muscle fiber areas compared to the control group [22]. Hence, these results suggest that early application of cold must be suppressed in muscle injuries. Considering recent contrasting findings and to the limited information on the effect of cryotherapy on muscle strength parameters, the objective of this work is to study the effect of 30-minute application of a cold pack to maximal 
force tetanic, maximal rate of contraction, fatigue time and fatigue in repeated contraction 1, 3- and 7-days post 2 hours of ischemia.

\section{Material and Methods}

\section{Animals and Experimental Groups}

An in-vivo experimental study was performed with forty-nine male adult Wistar rats (250-300 g). They were randomly divided into seven groups ( $\mathrm{n}=7$ per group): a group with no injury (control group); a group that was exposed to two-hours of ischemia, 1 day of reperfusion and no cold pack application; another group was exposed to two-hours of ischemia, 1 day of reperfusion and 30 minutes of cold pack application; one group was exposed to ischemia for 2 hours, 3 days of reperfusion and no cold pack application; one group was exposed to ischemia for 2 hours, 3 days of reperfusion and 30 minutes of cold pack application; another group was exposed to ischemia for 2 hours, 7 day reperfusion and no cold pack application; and finally, the last group was exposed to ischemia for 2 hours, 7 day reperfusion and 30 minutes of cold pack application. The order of assessment between groups was randomized. Animals were housed two per cage at the Animal Housing Facility of the Faculty of Health - Universidad ICESI, where temperature $\left(24^{\circ} \mathrm{C}\right)$, light ( $12 \mathrm{~h}$ on $-12 \mathrm{~h}$ off $)$, and humidity (55\%) were permanently controlled. Water and food were provided adlibitum and bedding material was placed in each cage. A half-time veterinarian and two full-time technicians made up the staff at the housing facility.

Cages were routinely cleaned, and the animals were checked on a daily basis to assess their condition. All animals were treated in accordance with the principles of the Guide to the Use of Experimental Animals of the Faculty of Health (Universidad Del Valle). All the experimental protocols were reviewed and approved by the Animal Care and Use Committee of the Universidad Del Valle.

\section{Experimental Protocols}

The rats were weighed and then sedated. They were first exposed for one minute to isoflurane $(100 \%)$ in a gas chamber (ISOFLUORANE USP $®$ - Baxter). Once they were anesthetized, the rats were deeply sedated with an intraperitoneal injection of pentobarbital sodium ( $6 \mathrm{mg} / 100 \mathrm{~g}$ body weight) (Pental®-INVET). Maintenance doses ( $2 \mathrm{mg} / 100 \mathrm{~g}$ body weight) were used as required. At the end of each experiment, an injection of pentobarbital sodium (18 mg/100 g body weight) was applied to induce euthanasia. The animals were kept under deep sedation while all the experimental protocols were performed.

\section{Ischemia-Reperfusion Injury}

The procedure for the generation of ischemia-reperfusion injury to the Soleus muscle has been previously described [13,2326]. Once anesthetized, rats were placed in prone decubitus position with the right inferior extremity in a pneumatic tourniquet above the knee joint. To achieve absolute ischemia the pressure of the tourniquet was increased to $350+/-10 \mathrm{mmHg}$. This pressure was controlled by a pressure transducer (Powerlab MLT1199 BP) during the two hours of ischemia. Finally, to achieve reperfusion tourniquet pressure was decreased to $0 \mathrm{~mm} \mathrm{Hg}$.

\section{Cryotherapy}

Direct transcutaneous application of a cold pack (Rubbermaid, Blue ice. $1.2 \times 2.3$ inches) was made on the lateral gastrocnemius muscle, simultaneously with the decrease in pressure of the pneumatic tourniquet. The temperature of the package ranged between $0.3-1.3^{\circ} \mathrm{C}$ and was monitored through a transducer (Powerlab MLT422/D) throughout the 30 minutes of application.

\section{Measurement of Intramuscular Temperature}

Temperature measurement of the Soleus muscle was performed in six experiments ( 4 with the application of a cold pack and 2 control groups), in which the rats were placed in prone position, an incision was realized in the lateral face of the right leg and a thermocouple (Powerlab MLT422/D) was placed below Soleus muscle. The room temperature was set at $24 \pm 1^{\circ} \mathrm{C}$ using an air conditioner.

\section{Measurement of Functional Parameters}

Preparation procedures for in-vivo functional measurements of Soleus muscle were previously described [12, 13, 26, 27]; rats were placed in prone position, the distal tendon was isolated, cut and tied with surgical thread and was assured to the force transducer (Powerlab MLT1030/A). Muscle temperature remained around $34+/-0.5^{\circ} \mathrm{C}$ through a cooling chamber connected to a bath of water circulation and measured by a digital thermometer. To maintain the muscle moistened throughout the experimental period an external perfusion of $15+/-2 \mathrm{ml} / \mathrm{min}$ was performed, using a ringer solution with values in $\mathrm{mM}$ of $135 \mathrm{NaCl} ; 5 \mathrm{KCl} ; 2 \mathrm{MgCl} 2$; 2CaCl2; 5 D-glucose and 10 HEPES. Before starting functional measurements, optimal length was determined by applying tetanic stimulation using a pulse of $0.2 \mathrm{~ms}$, frequency of $100 \mathrm{~Hz}$ with a voltage of 15 volts using a stimulator (Powerlab 16/35). Platinum electrodes were placed directly on the muscle belly.

\section{Tetanic Contraction:}

To generate tetanic contraction, stimulation was applied for 1 minute using $0.2 \mathrm{~ms}$ pulses with a frequency of $50 \mathrm{~Hz}$ and 15 volts. Functional parameters evaluated in tetanic tension were

a) maximum force or highest point of generation of force in tetanic tension from the baseline;

b) maximum contraction rate determined by the maximum derivative climb in tetanic contraction;

c) fatigue time determined by the time taken for a 30\% drop of the maximum force to occur.

\section{Repeated Tetanic Contractions:}

To determine the drop-in force in repeated stimulation, the stimulus was applied using 15 pulses of $0.2 \mathrm{~ms}$ to 15 volts with a frequency of $100 \mathrm{~Hz}$, the interval being $1.39 \mathrm{~s}$ during $5 \mathrm{~min}$ for a total of 215 contractions [28]. This protocol analyzed the drop of force during stimulation period.

\section{Statistical Analysis:}

The data were initially tested for normality using the Shapiro Wilk statistics. This assumption was validated, and the data are 
presented as the means \pm s.e.m. One-way ANOVA with Bonferroni posthoc test was then used to test for significance between exper- imental groups. Differences between the control and experimental groups were analyzed using a statistical significance of $\mathrm{p}<0.05$.

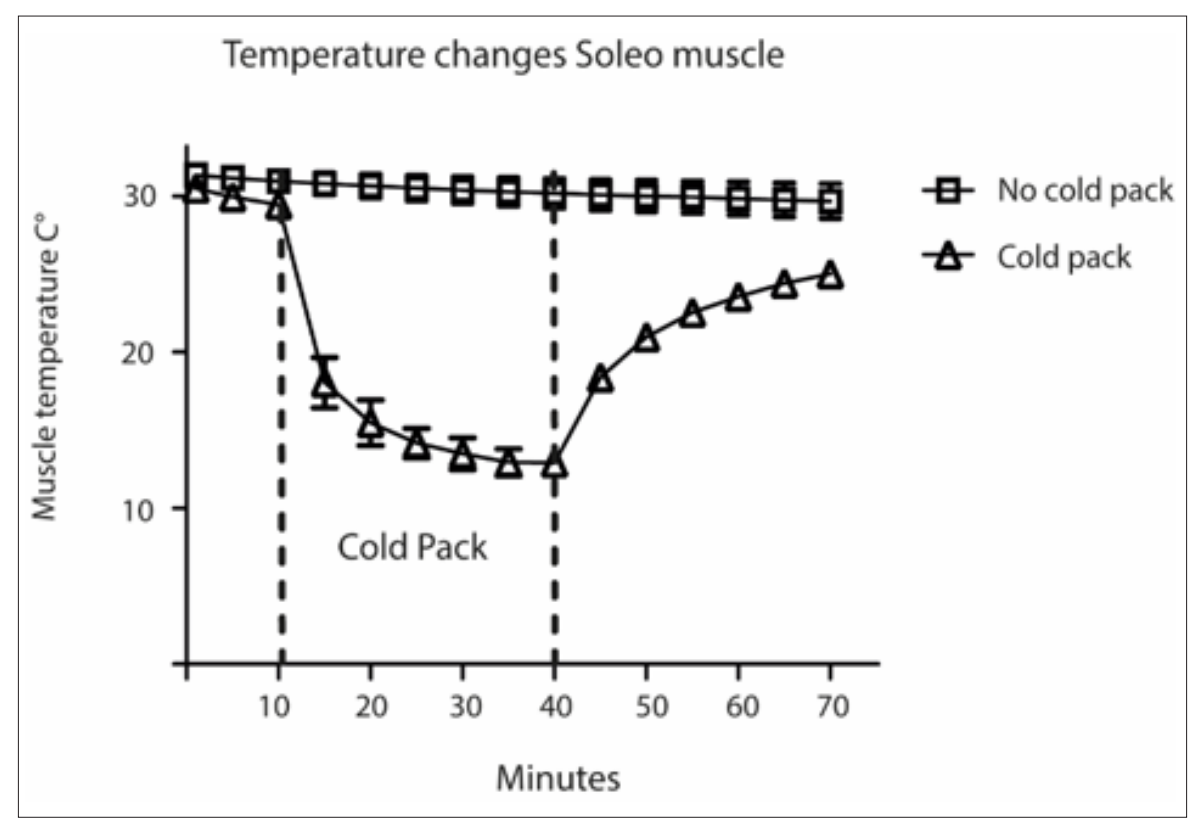

Figure 1: Temperature changes Soleo muscle during cold pack application.

\section{Results}

\section{Cold Pack Reduces in $43 \%$ the Temperature of the Soleus Muscle}

The temperature of Soleus muscle before the application of the cold pack was $29.4 \pm 0.3^{\circ} \mathrm{C}$. During the transcutaneous application of the cold pack, the temperature in the Soleus muscle decreased in a curvilinear manner throughout the thirty minutes, presenting its largest decrease of temperature in the first five minutes, reducing the temperature to $18^{\circ} \pm 1.6^{\circ} \mathrm{C}$. At the end of the 30 minutes, the temperature had decreased to $12.9 \pm 0.7^{\circ} \mathrm{C}$. After removal of the cold pack, the temperature began to rise, increasing to $25 \pm 0.3^{\circ} \mathrm{C}$ after thirty minutes, without reaching control values within the assessment period (Figure 1).

\section{The Groups without Cold Pack Present the Greatest Fall} of the Force on the Third-Day Post-Ischemia

Maximum tetanic force in the control group was on average 84g. After 1-day ischemia, the without cold pack group presented a mean force 49,8 $\mathrm{g}$ and with cold pack presented a mean force of 60 g with no significant changes compared to the control group. After 3 days, the group without the cold pack presented a mean force of 42 g, representing a 50\% drop in respect to the control group $(\mathrm{p}<0.05)$, the group with cold pack application had a mean force of $62 \mathrm{~g}$, and thus there were no significant differences in respect to the control group. After 7 days, the mean tetanic force corresponded to 44 and $52 \mathrm{~g}$ in the group without and with the cold pack, representing a $48 \%$ and $38 \%$ drop compared to the control group, respectively (Figure 2A-C).
The Group with Cold Pack present Decreased in the Maximum Contraction Rate One Day and the Group without Cold Pack Decreased One and Three-Day PostIschemia:

The maximum contraction rate in the control group had an average of $712 \mathrm{~g} / \mathrm{s}$. After 1 day of ischemia, maximum contraction rate in both experimental groups had an average of 490 and 399 g/s representing a $32 \%$ and $44 \%$ fall compared to the control group ( $\mathrm{p}<0.01$ ). After 3 days, the value rose to $567 \mathrm{~g} / \mathrm{s}$ for the group with a cold pack and the group no cold pack reduced $441 \mathrm{~g} / \mathrm{s}$ thus this is a significant difference with the control group $(\mathrm{p}<0.01)$. After 7 days, values increased more reaching 511 and $587 \mathrm{~g} / \mathrm{s}$ for the group with no cold pack and the group with the cold pack respectively, displaying important differences between the control and experimental groups (Figure 2D).

\section{The Group without Cold Pack Present $90 \%$ of Decreased the Fatigue Time Three Days Post-Ischemia}

Fatigue time in the control group presented an average of $57 \mathrm{~s}$. After 1 day of ischemia, this time decreased to $24 \mathrm{~s}$ for the two experimental groups, representing a decline of $49 \%$ compared to the control group $(\mathrm{p}<0.001)$. After 3 days, fatigue time was $6 \mathrm{~s}$ in the group with no cold pack and $18 \mathrm{~s}$ in the group with a cold pack, representing a decrease of $90 \%$ and $69 \%$ in respect to the control group ( $\mathrm{p}<0.01$ ). After 7 days, fatigue time values reached up to 34 $\mathrm{s}$ and $29 \mathrm{~s}$ for the group without a cold pack and with cold pack application, respectively. Anyhow, it still represented a 40\% drop compared to the control group $(\mathrm{p}<0.01)$ (Figure 2E). 
A

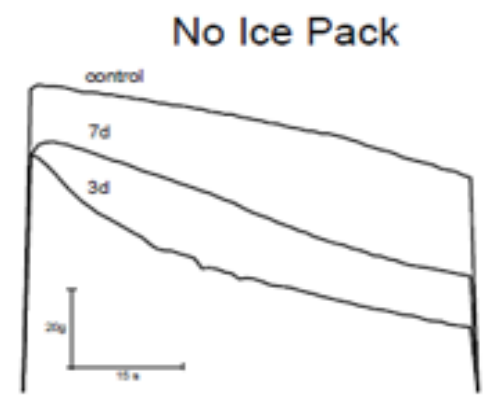

C

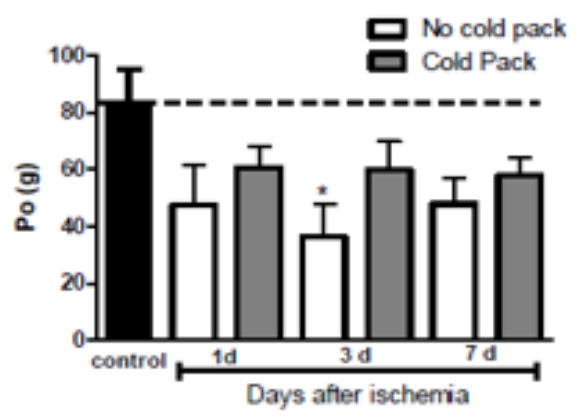

B

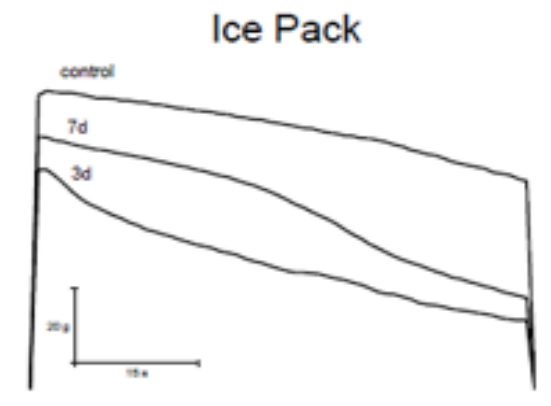

$\mathrm{D}$

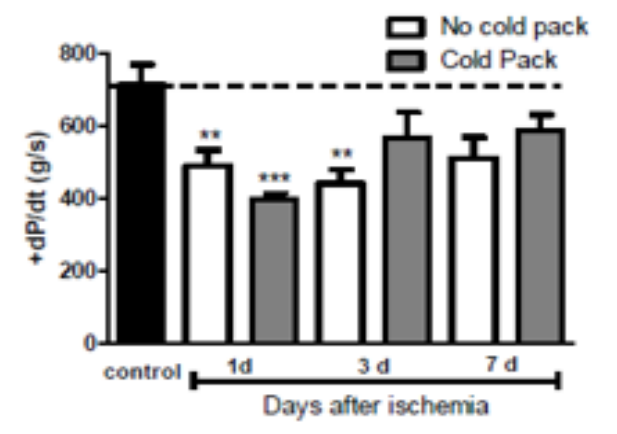

E

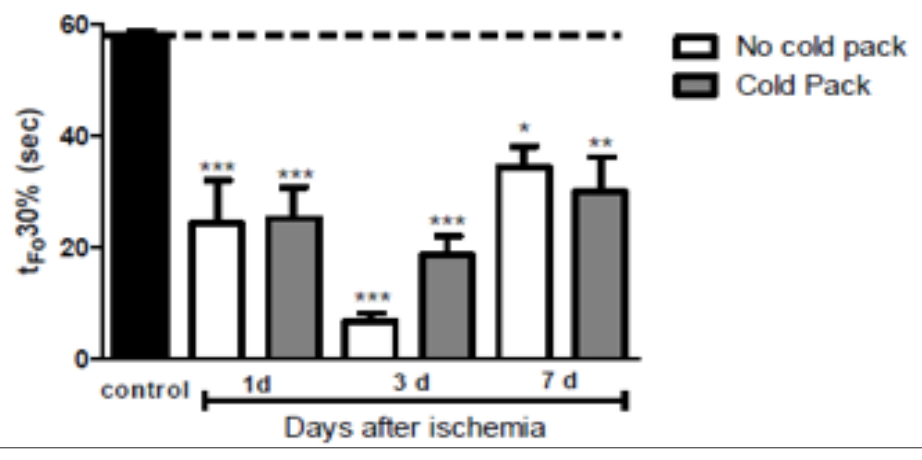

Figure 2: Tetanic force are compared in control, 3 and 7 days post ischemia without (A) and with cold pack (B). Chronological changes in maximal tetanic force (C), maximal rate of contraction (D) and fatigue time (E). The horizontal axis plots the group without injury and time in days of the ischemic injury groups (1, 3 and 7 days). The values presented are the mean \pm SE. Black squares, group without injuries; black squeres group without cold pack; gray squares, cold pack group. Significant differences with the group without injury and with and without cold pack, ${ }^{*}: P<0.05 ;{ }^{* *}: \mathrm{p}<0.01{ }^{* * *}: \mathrm{p}<0.001$.

The Group without Cold Pack present more Decreased in Fatigue in Repeated Contractions after 1- and 3-Days Post-Injury

Fatigue produced by repeated tetanic contractions presented a decrease in force in the control group equivalent to $30 \%$ in the $1^{\text {st }}$ minute, $49 \%$ in the $2^{\text {nd }}$ minute, $59 \%$ in the $3^{\text {rd }}$ minute, $63 \%$ in the 4 th minute and $66 \%$ in the $5^{\text {th }}$ minute (Figures 3A \& 3B). After 1 days, there are significant differences between the experimental groups.
The drop in maximum force reached $50 \%$ during the $1^{\text {st }}$ minute, thin being $20 \%$ lower than control. After three days the strength reduction in the first minute is maintained for both experimental groups but at one minute the strength drop in the group without a larger, with significant differences between the group are cold pack and the control groups and with a cold pack. (Figure 3C). After 7 days, there is no difference between the three experimental groups (Figure 3D). 

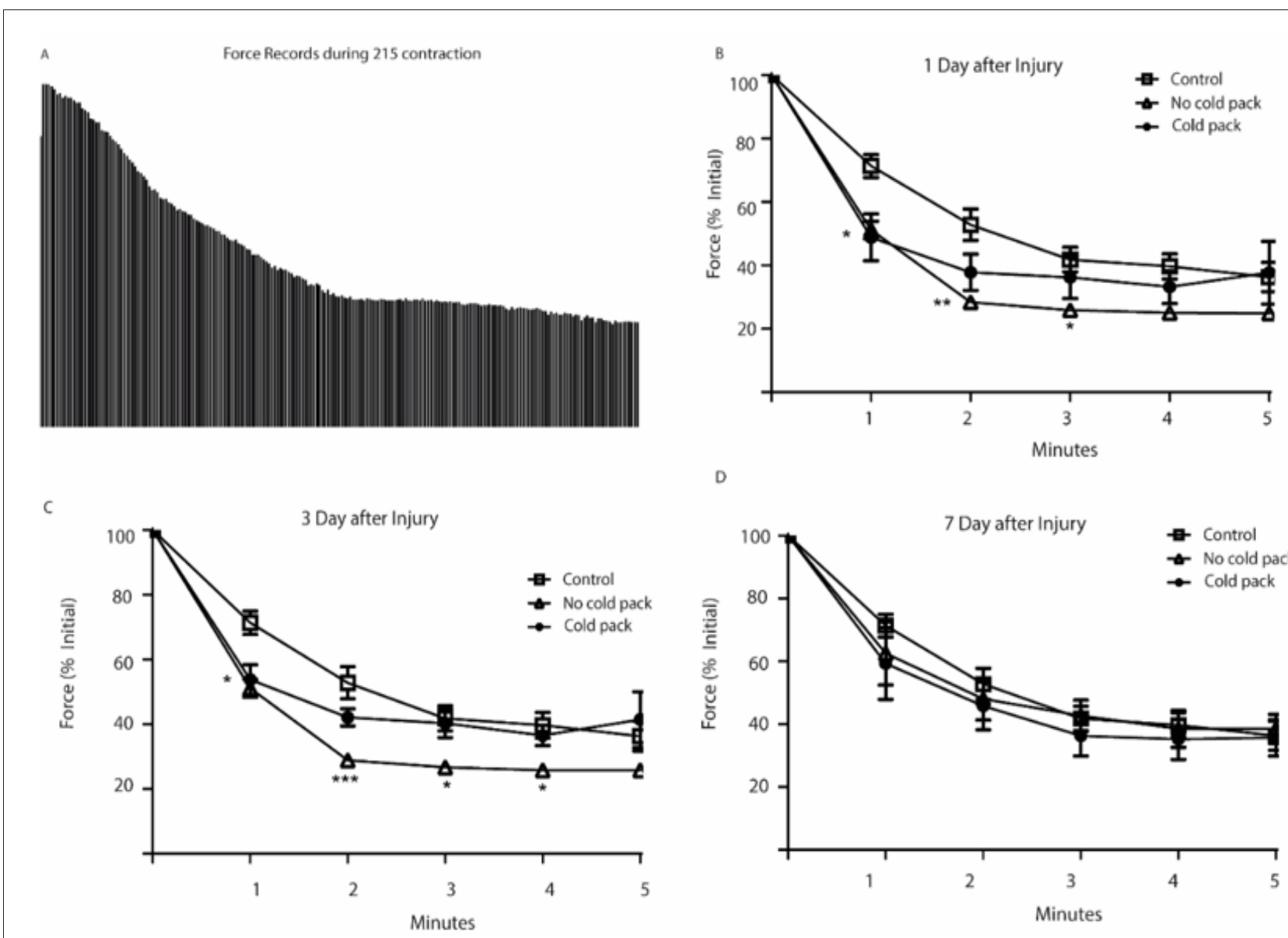

D

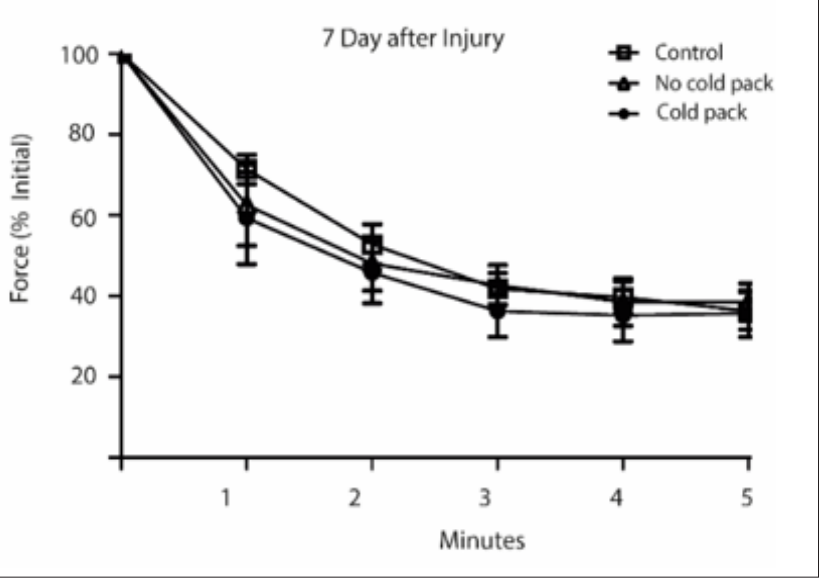

Figure 3: Representation of 215 tetanic contractions for 5 minutes for the group with no injury (A); percentage of force drop 1-day post-ischemia injury (B), three days after ischemic injury (C) and 7 days after ischemic injury (D). White squares group without injuries; white triangles group without cold pack; Black circles, cold pack group. + Significant differences with the group without injury, $\mathrm{P}<0.05$.

\section{Discussion}

\section{Changes in Muscular Temperature}

The muscular temperature drop during the 30 minutes of cold pack application was of $17^{\circ} \mathrm{C}$ (from $29^{\circ} \mathrm{C}$ to $12^{\circ} \mathrm{C}$ ). These results agree with the study done on ankle dorsiflexor muscles in humans (8 $\mathrm{mm}$ deep), in which an $18^{\circ} \mathrm{C}$ drop was reported during the application of cryotherapy at $0^{\circ} \mathrm{C}$ for 30 minutes [29]. This study also showed that in order to achieve such a reduction it is necessary to maintain the temperature of the cold pack at $0^{\circ} \mathrm{C}$. Therefore, it is very important to monitor. the temperature during clinical application, as was done in this study. Another factor to consider with these results is that it cannot be applied to deep muscles (more than $18 \mathrm{~mm}$ ), since reductions in temperature are around 6 to $8^{\circ} \mathrm{C}$ after 30 minutes of application [30,31,29]. However, it has been reported that to reduce the temperature on deep muscles to $8^{\circ} \mathrm{C}$, an increase in time to about 90 minutes is required 34. These findings suggest that in cryotherapy, the time of application depends on the depth of the tissue of interest.

\section{Functional Parameters}

Data concerning maximum force, the maximum rate of contraction, fatigue time and fatigue in repeated contractions show that three days' post-ischemia the group without cold pack present significant differences with the control group. The group without a cold pack in the three days is the only have present significantly differences with the control group with 57\% reduction on force while the group cold pack the maximum reduction of force is the $32 \%$. The maximum contraction rate, determined by the maximum slope of the climb in tetanic contraction, represents the highest velocity of calcium liberation from the sarcoplasmic reticulum, binding of calcium with troponin $\mathrm{C}$ and actin-myosin union $[32,33]$. It was observed that two hours of ischemia only caused a $21 \%$ decrease, which gradually increased from day 3 to day 7 after injury. Fatigue time was the most affected functional parameter during the two hours of ischemia in both experimental groups, specifically when a tetanic contraction was sustained for 1 minute. Similarly, maximum tetanic force dropped to a maximum of 35\%, maximum contraction rate to $22 \%$, while fatigue time changed by $71 \%$. 
Fatigue during a sustained tetanic contraction is also called high-frequency fatigue and its main characteristic is the absence of blood perfusion, this being a predisposing factor for the utilization of anaerobic energy sources in the muscle for ATP production [34]. A factor to analyze in future research is the fact that in the thirdday post-ischemia both experimental groups presented the largest fatigue and in the seventh day, it presented a fall of $40 \%$, is this parameter the one with the lowest recovery in tetanic contraction. The rate of fatigue in repeated stimulations is another point of great interest. This, unlike the fatigue time, is a natural pattern of the skeletal muscles daily activities such as walking, running or breathing [34] It is known as prolonged low-frequency force depression and in studies of muscles with a greater predominance of oxidative fibers stimulated at $100 \mathrm{HZ}$ for 5 minutes as performed in this study, the main cause of a fall of force is due to a decrease in the total calcium content $[34,32]$. This suggests that in the 2 hours ischemia process, with and without the application of the cold pack there is no change in the total calcium content in comparison with the group without injury. This means that although muscle strength is decreased (maximum tetanic force), changes in the amounts of total calcium are like the group without injury when assessed 1- and 7-days post-injury. Surprisingly, 3 days post-ischemia both experimental groups shows a fall 15\% higher than the control group, suggesting that during this day, the highest muscle fatigability was present.

\section{Conclusion}

Previously the effects of ischemia-reperfusion in the skeletal muscle in the histological parameters are reported changes in muscle fiber area, edema and inflammatory [8] and the use of cold pack minimizes the impact of injury in muscle structure [1,20-22] but we also found that cryotherapy placed 30 minutes during reperfusion accelerates functional recovery on the third day after ischemia. The group without cold pack present decreased significantly on the maximum force, maximum contraction rate and fatigue time on day 1 and 3 comparatives with the control group while the group with cold pack present significant differences only the first day. These results suggest that for ischemia-reperfusion injuries such surgical situations involving the use of pneumatic tourniquets, the use of cold pack modifies histological patterns and accelerates the recovery in functional parameters.

\section{Acknowledgment}

This work was supported by Pontificia Universidad Javeriana Cali. Grant number 020100277. The author thanks, Osvaldo Delbono for fatigue in repeated contractions protocol and William Ocampo for your laboratory. The author declares that there are no conflicts of interest.

\section{References}

1. Puntel GO, Carvalho NR, Amaral GP, Lobato LD, Silveira SO, et al. (2011) Therapeutic cold: An effective kind to modulate the oxidative damage resulting of a skeletal muscle contusion. Free Radic Res 45(2): 125-138.

2. Agrawal K, Eberhardt RT (2015) Contemporary medical management of peripheral arterial disease: a focus on risk reduction and symptom relief for intermittent claudication. Cardiol Clin 33(1): 111-137.
3. Pipinos II, Judge A R, Selsby JT, Zhu Z, Swanson SA, Nella AA, Dodd SL (2008) The myopathy of peripheral arterial occlusive disease: Part 2. Oxidative stress, neuropathy, and shift in muscle fiber type. Vasc Endovascular Surg 42(2): 101-112.

4. Khanna A, Cowled PA, Fitridge RA (2005) Nitric oxide and skeletal muscle reperfusion injury: current controversies (research review). J Surg Res 128(1): 98-107.

5. Tran TP, Tu H, Pipinos II, Muelleman RL, Albadawi H, et al. (2011) Tourniquet-induced acute ischemia-reperfusion injury in mouse skeletal muscles: Involvement of superoxide. Eur J Pharmacol 650(1): 328-334.

6. Wang W Z, Baynosa RC, Zamboni WA (2011) Therapeutic interventions against reperfusion injury in skeletal muscle. J Surg Res 171(1): 175182.

7. Blaisdell FW (2002) The pathophysiology of skeletal muscle ischemia and the reperfusion syndrome: a review. Cardiovasc Surg 10(6): 620630 .

8. Carmo Araujo EM, Dal Pai Silva M, Dal Pai V, Cecchini R, Anjos Ferreira AL (2007) Ischaemia and reperfusion effects on skeletal muscle tissue: morphological and histochemical studies. Int J Exp Pathol 88(3): 147154.

9. Gillani S, Cao J, Suzuki T, Hak DJ (2012) The effect of ischemia reperfusion injury on skeletal muscle. Injury 43(6): 670-675.

10. Kalogeris T, Baines CP, Krenz M, Korthuis RJ (2012) Cell biology of ischemia/reperfusion injury. Int Rev Cell Mol Biol 298: 229-317.

11. Walters TJ, Kragh JF, Baer DG (2008) Influence of fiber-type composition on recovery from tourniquet-induced skeletal muscle ischemia-reperfusion injury. Appl Physiol Nutr Metab 33(2): 272-281.

12. Carvalho AJ, McKee NH, Green HJ (1997) Metabolic and contractile responses of fast and slow twitch rat skeletal muscles to ischemia and reperfusion. Plast Reconstr Surg 99(1): 163-171.

13. Morales C, Fierro L (2017) Ischemia Increases the Twitch Latent Period in the Soleus and Extensor Carpi Radialis Longus Muscles from Adult Rats. J Invest Surg 30(5): 303-313.

14. Woitaske MD, McCarter RJ (1998) Effects of fiber type on ischemia-reperfusion injury in mouse skeletal muscle. Plast Reconstr Surg 102(6): 2052-2063.

15. Brukner P, Khan K (2007) Clinical Sports Medicine: McGraw-Hill.

16. Howatson G, Gaze D, van Someren K A (2005) The efficacy of ice massage in the treatment of exercise-induced muscle damage. Scand J Med Sci Sports 15(6): 416-422.

17. Mustalampi S, Ylinen J, Kautiainen H, Weir A, Hakkinen A (2012) Acute effects of cold pack on mechanical properties of the quadriceps muscle in healthy subjects. Phys Ther Sport 13(4) 265-269.

18. Nadler SF, Weingand K, Kruse RJ (2004) The physiologic basis and clinical applications of cryotherapy and thermotherapy for the pain practitioner. Pain Physician 7(3): 395-399.

19. White GE, Wells GD (2013) Cold-water immersion and other forms of cryotherapy: physiological changes potentially affecting recovery from high-intensity exercise. Extrem Physiol Med 2(1): 26.

20. Schaser KD, Disch AC, Stover JF, Lauffer A, Bail HJ, et al. (2007) Prolonged superficial local cryotherapy attenuates microcirculatory impairment, regional inflammation, and muscle necrosis after closed soft tissue injury in rats. Am J Sports Med 35(1): 93-102.

21. Vieira Ramos G, Pinheiro CM, Messa SP, Delfino GB, Marqueti Rde C, et al. (2016) Cryotherapy Reduces Inflammatory Response Without Altering Muscle Regeneration Process and Extracellular Matrix Remodeling of Rat Muscle. Sci Rep 6: 18525.

22. Takagi R, Fujita N, Arakawa T, Kawada S, Ishii N, et al. (2011) Influence of icing on muscle regeneration after crush injury to skeletal muscles in rats. J Appl Physiol 110(2): 382-388. 
23. Conrad MF, Albadawi H, Stone DH, Crawford RS, Entabi F, et al. (2006) Local administration of the Poly ADP-Ribose Polymerase (PARP) inhibitor, PJ34 during hindlimb ischemia modulates skeletal muscle reperfusion injury. J Surg Res 135(2): 233-237.

24. Chen XK, Rathbone CR, Walters TJ (2011) Treatment of tourniquet-induced ischemia reperfusion injury with muscle progenitor cells. J Surg Res 170(1): e65-73.

25. Tsai YL, Hou CW, Liao YH, Chen CY, Lin FC, et al. (2006) Exercise training exacerbates tourniquet ischemia-induced decreases in GLUT4 expression and muscle atrophy in rats. Life Sci 78(25): 2953-2959.

26. Walters TJ, Kragh JF, Kauvar DS, Baer DG (2008) The combined influence of hemorrhage and tourniquet application on the recovery of muscle function in rats. J Orthop Trauma 22(1): 47-51.

27. Winkler T, von Roth P, Matziolis G, Schumann MR, Hahn S, et al. (2011) Time course of skeletal muscle regeneration after severe trauma. Acta Orthop 82(1): 102-111.

28. Gonzalez E, Delbono 0 (2001) Age-dependent fatigue in single intact fast- and slow fibers from mouse EDL and soleus skeletal muscles. Mech Ageing Dev 122(10): 1019-1032.

\section{ISSN: 2574-1241}

DOI: 10.26717/BJSTR.2018.11.002041

Camilo Morales Jiménez. Biomed J Sci \& Tech Res

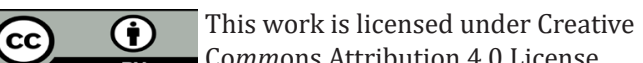

Submission Link: https://biomedres.us/submit-manuscript.php
29. Yanagisawa O, Homma T, Okuwaki T, Shimao D, Takahashi H (2007) Effects of cooling on human skin and skeletal muscle. Eur J Appl Physiol $100(6): 737-745$.

30. Dykstra JH, Hill HM, Miller MG, Cheatham CC, Michael TJ, et al. (2009) Comparisons of cubed ice, crushed ice, and wetted ice on intramuscular and surface temperature changes. J Athl Train 44(2): 136-141.

31. Merrick MA, Jutte LS, Smith ME (2003) Cold Modalities with Different Thermodynamic Properties Produce Different Surface and Intramuscular Temperatures. J Athl Train 38(1): 28-33.

32. Gurke L, Marx A, Sutter PM, Stierli P, Harder F, et al. (2000) Function of fast- and slow-twitch rat skeletal muscle following ischemia and reperfusion at different intramuscular temperatures. Eur Surg Res 32(3): 135-141.

33. Rome LC (2006) Design and function of superfast muscles: new insights into the physiology of skeletal muscle. Annu Rev Physiol 68: 193-221.

34. Allen DG, Lamb GD, Westerblad H (2008) Skeletal muscle fatigue: cellular mechanisms. Physiol Rev 88(1): 287-332.

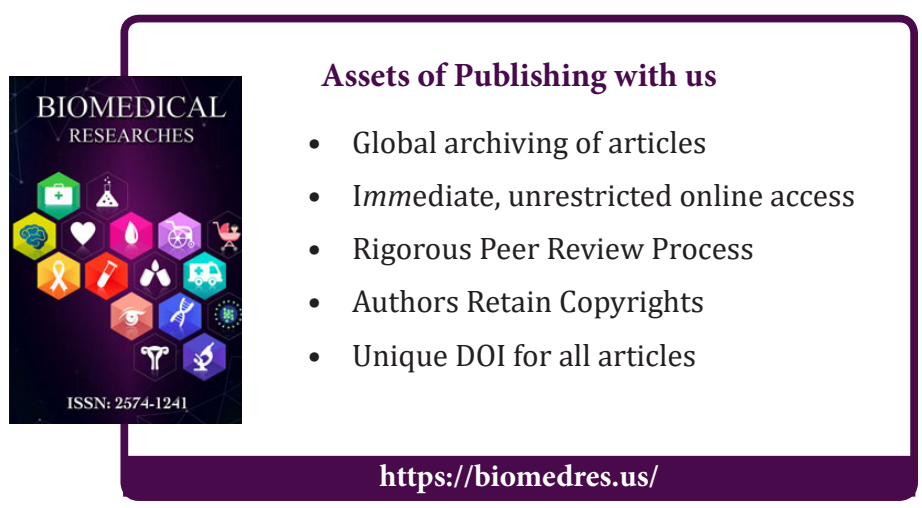

